\title{
Transcriptome sequencing and metabolite analysis for revealing the blue flower formation in waterlily
}

\author{
Qian $\mathrm{Wu}^{1,2}$, Jie Wu ${ }^{1,2}$, Shan-Shan $\mathrm{Li}^{1 *}$, Hui-Jin Zhang ${ }^{1}$, Cheng-Yong Feng ${ }^{1,2}$, Dan-Dan Yin ${ }^{1,2}$, Ru-Yan Wu ${ }^{1,3}$ \\ and Liang-Sheng Wang ${ }^{1 *}$
}

\begin{abstract}
Background: Waterlily (Nymphaea spp.), a perennial herbaceous aquatic plant, is divided into two ecological groups: hardy waterlily and tropical waterlily. Although the hardy waterlily has no attractive blue flower cultivar, its adaptability is stronger than tropical waterlily because it can survive a cold winter. Thus, breeding hardy waterlily with real blue flowers has become an important target for breeders. Molecular breeding may be a useful way. However, molecular studies on waterlily are limited due to the lack of sequence data.

Results: In this study, six cDNA libraries generated from the petals of two different coloring stages of blue tropical waterlily cultivar Nymphaea 'King of Siam' were sequenced using the Illumina HiSeq ${ }^{\text {TM }} 2500$ platform. Each library produced no less than $5.65 \mathrm{~Gb}$ clean reads. Subsequently, de novo assembly generated 112,485 unigenes, including 26,206 unigenes annotated to seven public protein databases. Then, 127 unigenes could be identified as putative homologues of color-related genes in other species, including 28 up-regulated and 5 down-regulated unigenes. In petals, 16 flavonoids ( 4 anthocyanins and 12 flavonols) were detected in different contents during the color development due to the different expression levels of color-related genes, and four flavonols were detected in waterlily for the first time. Furthermore, UA3GTs were selected as the most important candidates involved in the flavonoid metabolic pathway, UA3GTs induced blue petal color formation in Nymphaea 'King of Siam'.
\end{abstract}

Conclusions: This study will improve our understanding of the molecular mechanism of blue flowers in waterlily and provide the basis for molecular breeding of blue hardy waterlily cultivars.

Keywords: Waterlily, Transcriptome, Anthocyanin, Flavonoid, Blue color, UAGT

\section{Background}

Flower color is one of the most important characters of ornamental plants, which determined by many factors. However, the type and content of anthocyanins are generally the primary factor $[1,2]$. Although anthocyanins have various structures, there are only seven common chromophore forms (aglycones) of anthocyanins: cyanidin, pelargonidin, delphinidin, peonidin, petunidin, malvidin, and hirsutidin $[3,4]$. Among a wide variety of flower colors, blue arises primarily from anthocyanins, which are specifically derived from delphinidin. Breeders

\footnotetext{
*Correspondence: shshli@ibcas.ac.cn; wanglsh@ibcas.ac.cn

'Key Laboratory of Plant Resources and Beijing Botanical Garden, Institute of

Botany, Chinese Academy of Sciences, Beijing 100093, China

Full list of author information is available at the end of the article
}

always devote to breeding blue cultivars because it is difficult to obtain real blue flowers in many floricultural plants, such as carnation, chrysanthemum, rose, lotus, and hardy waterlily $[5,6]$. Traditional breeding methods have many constraints, such as sterility of species and uncontrollable traits, which can be circumvented by genetic engineering [7].

Waterlily (Nymphaea spp.), a perennial herbaceous aquatic plant, occurs almost worldwide and has 45-50 species in five subgenera: Anecphya (7-10 species), Brachyceras (14-16 species), Hydrocallis (14 species), Lotos (2-3 species), and Nymphaea (8 species) [8]. It is called subaqueous nymph. Waterlily has played an obvious role in the history of different cultures, such as ancient Egypt, India, and Thailand. In addition to its ornamental 
value, waterlily can be used to prepare foods and drinks [9]. It also has diverse biological activities, including antibacterial [10], antimicrobial [11], anti-inflammatory [12], antidiabetic [13, 14], antiradiation [15], antianxiety [16], and antihepatotoxic activities [17]. Waterlily is also divided into two ecological groups, namely, hardy waterlily and tropical waterlily [18]. Hardy waterlily can survive a cold winter and its colors range from dark red to white. Tropical waterlily cannot survive a cold winter in the outdoor pond in the region with the latitude more than 25 degrees, so it has a smaller distribution. However, tropical waterlily is more favorable and it has special flower colors with blue, violet and bluish purple [19]. Thus, breeding hardy waterlily with blue flowers has become an important target for breeders. Although conventional breeding methods may achieve this goal, a truly blue petal color of hardy waterlily has not been reported yet [20].

Molecular breeding provides a new way to obtain blue hardy waterlily. Therefore, it is necessary to understand the molecular mechanism of blue characteristic formation in waterlily and identify related key genes for the purpose of modifying flower color in hardy waterlily by genetic engineering in breeding blue hardy waterlily in the future. The next-generation sequencing technology, RNA sequencing (RNA-Seq), has provided unique insights into the molecular mechanism for non-model organisms without a reference genome [21-23]. As a transcriptome profiling approach utilizing deep-sequencing technologies, RNA-Seq provided the more precise measurements of transcripts and their isoforms than other methods [24].

In the study, the first RNA-Seq project for the petals of two different coloring stages of tropical waterlily cultivar Nymphaea 'King of Siam' was performed using Illumina HiSeq $^{\text {ma }} 2500$ platform. Qualitative and quantitative analysis of flavonoids were performed with I-Class ultra-high performance liquid chromatography/Xevo triple-quadrupole mass spectrometry (I-Class UPLC/Xevo TQ MS) technology and the expression patterns of differentially expressed genes (DEGs) identified in the flavonoid biosynthetic pathway were analyzed using real-time quantitative polymerase chain reaction (RT-qPCR). The above analysis results can reveal the biochemical and molecular mechanisms of blue color formation in Nymphaea 'King of Siam' and provide the basis for breeding blue hardy waterlily cultivars. Furthermore, this study will improve our understanding of the molecular mechanism of blue flowers and provide the evidence for molecular breeding theory concerning the basis of flower color modifications in ornamental plants.

\section{Methods}

\section{Plant materials}

The petals of tropical waterlily cultivar Nymphaea 'King of Siam' were selected as the experimental materials.
This cultivar has petaloid stamens in deep blue-violet (RHSCC: 92A) color (S6 in Fig. 1). The samples were collected in six different coloring stages in August 2014 at Beijing Botanical Garden, Institute of Botany, the Chinese Academy of Sciences, Beijing, China. In S1, petals were colorless; in S2, the outer petals were partially colorated; in S3, the outer petals were fully colorated, while inner petals were colorless; in S4, the outer petals were fully colorated, while inner petals were partially colorated; in S5, petals were fully colorated; S6 is the first day of blooming (Fig. 1). All the collected petal samples were immediately frozen in liquid nitrogen and stored at $-80{ }^{\circ} \mathrm{C}$ for RNA extraction and flavonoid analysis.

\section{Measurement of flavonoids}

The extraction of flavonoids was performed according to the previously described method with some modifications [25]. Approximately $0.2 \mathrm{~g}$ of frozen petals were pulverized in liquid nitrogen, extracted with $1 \mathrm{~mL}$ of extracting solution (99.8: 0.2, v/v, methanol: formic acid) in a test tube, sonicated with KQ-500DE ultrasonic cleaner (Ultrasonic instruments, Jiangsu Kunshan, China) at $20{ }^{\circ} \mathrm{C}$ for $20 \mathrm{~min}$, and then centrifuged in SIGMA 3 K30 (SIGMA centrifuger, Germany) with

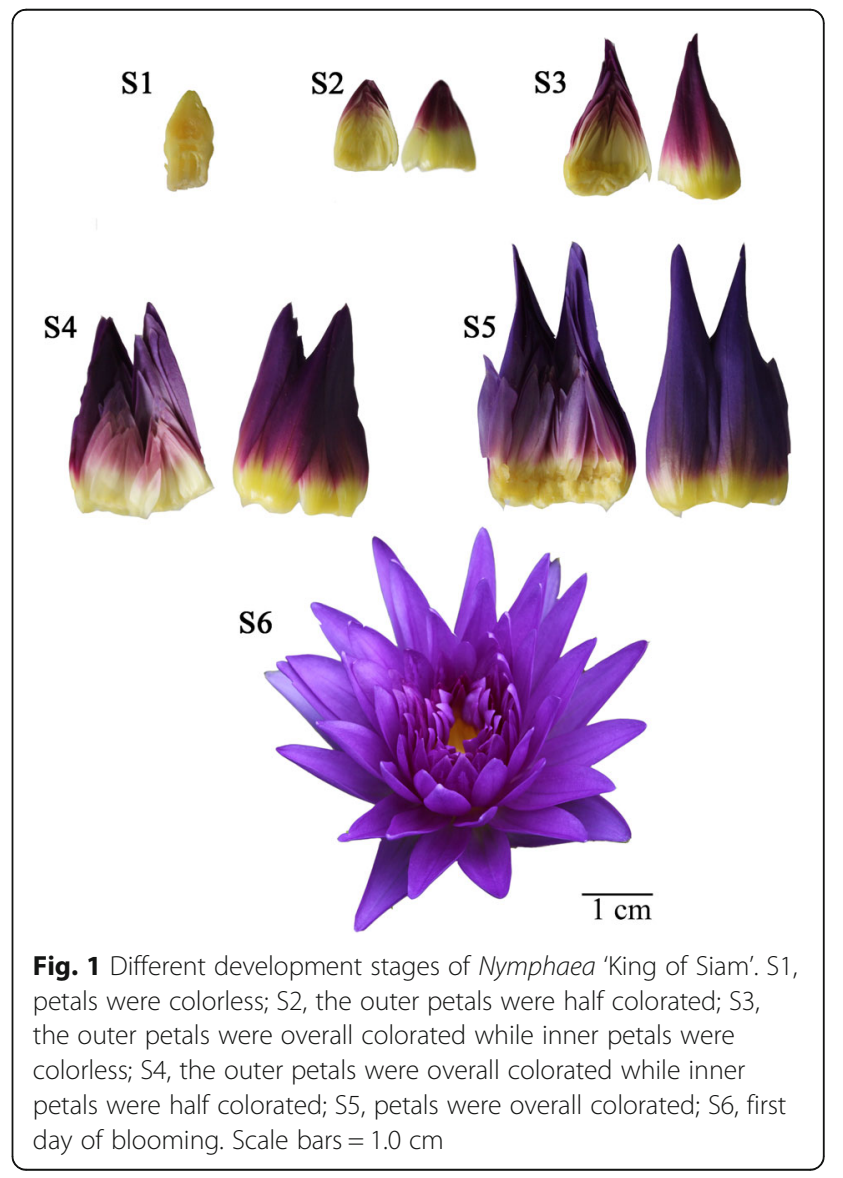


$10000 \mathrm{~g}$ for $10 \mathrm{~min}$. The supernatants were collected into fresh tubes. We repeated the above operation for the second time. All extracts were combined and filtered through $0.22 \mu \mathrm{m}$ reinforced nylon membrane filters (Shanghai ANPEL, Shanghai, China) before the I-Class ultra-high performance liquid chromatography (I-Class UPLC) (Waters, USA) analysis. Three analysis replicates were made for each sample.

I-Class ultra-high performance liquid chromatography/ Xevo triple-quadrupole mass spectrometry (I-Class UPLC/Xevo TQ MS) was used for qualitative analysis. The liquid chromatograph was equipped with an ACQUITY UPLC HSS C18 column $(2.1 \mathrm{~mm} \times 100 \mathrm{~mm}$, $1.7 \mu \mathrm{m}$ ) (Waters, USA). Eluent A was $1 \%$ formic acid aqueous solution and Eluent $B$ was acetonitrile. The following gradient profile was used: $5 \% \mathrm{~B}$ at $0 \mathrm{~min}, 45 \% \mathrm{~B}$ at $6 \mathrm{~min}, 90 \% \mathrm{~B}$ at $7 \mathrm{~min}, 10 \% \mathrm{~B}$ at $7.1 \mathrm{~min}$, and $10 \% \mathrm{~B}$ at $10 \mathrm{~min}$. The flow rate was $0.2 \mathrm{~mL} / \mathrm{min}$ and the injected volume was $5 \mu \mathrm{L}$. Column temperature was maintained at $35^{\circ} \mathrm{C}$ and sample temperature was $10{ }^{\circ} \mathrm{C}$. Chromatograms of anthocyanins and other flavonoids were acquired at $525 \mathrm{~nm}$ and $350 \mathrm{~nm}$, respectively. The following analysis conditions of mass spectrometry were adopted: the positive-ion (PI) mode for anthocyanins and negative-ion (NI) mode for other flavonoids; capillary voltage of $3.00 \mathrm{kV}$; cone voltage of $20 \mathrm{~V}$ for PI mode and cone voltage of $70 \mathrm{~V}$ for NI mode; desolvation gas $\left(\mathrm{N}_{2}\right)$ flow of $800 \mathrm{~L} / \mathrm{h}$; cone gas flow of $50 \mathrm{~L} / \mathrm{h}$; collision gas flow of $0.12 \mathrm{~mL} / \mathrm{min}$; collision energy of $10 \mathrm{eV}$; the desolvation temperature of $400{ }^{\circ} \mathrm{C}$; source temperature of $150{ }^{\circ} \mathrm{C}$; and the scanning range of $100-1000(\mathrm{~m} / \mathrm{z})$ units.

The contents of anthocyanins and other flavonoids were calculated in milligrams per $100 \mathrm{~g}$ fresh weight. Cyanidin 3-O-glucoside (Cy3Glc) and rutin were used as quantification standards. Mean values and SDs were calculated from three biological replicates.

\section{RNA isolation, library construction and RNA-Seq}

Total RNA was isolated with RN40-EASYspin plant microRNA kit (Aidlab Biotechnology Co., Ltd., Beijing, China), characterized on a $1 \%$ agarose gel and examined with a NanoDrop 2000 UV-vis spectrophotometer (Thermo Fisher Scientific Inc., Waltham, MA, USA). Agilent 2100 Bioanalyzer (Agilent Technologies Co. Ltd., Santa Clara, CA, USA) was used to assess RIN (RNA integrity number) of each sample. The construction of the libraries and RNA-Seq were performed by Biomarker Biotechnology Corporation (Beijing, China). Firstly, mRNA was enriched using Oligo (dT) attached to magnetic beads. Then mRNA was broken into short fragments through the addition of fragmentation buffer. Taking these short fragments as templates, the firststrand cDNA was synthesized with random hexamers. Buffer, dNTPs, RNase $\mathrm{H}$, and DNA polymerase were mixed to synthesize second-strand cDNA. The cDNAs were purified with AMPure XP beads and then subjected to end-repair. To repair cDNAs, an 'A' base was inserted at the $3^{\prime}$ end. After that, adaptors were connected to these cDNA fragments to select different fragments. PCR amplification was performed to enrich cDNA library. Agilent 2100 Bioanaylzer and Qubit ${ }^{\circ}$ Fluorometer (Thermo Fisher Scientific Inc., Waltham, MA, USA) were used to assess the quality of cDNA library. At last, the six libraries were sequenced by using an Illumina HiSeq $^{\text {mi }} 2500$ platform with 125 bp paired-end reads.

\section{De novo assembly and functional annotation}

Firstly, after removing the adapter sequences, low quality sequences, unknown reads ( $\mathrm{N}$ percentage $>10 \%$ ) and ribosomes RNA from raw reads, the rest of raw reads called clean reads were obtained. Then, clean reads were used for transcriptome de novo assembly using trinity platform (http://trinityrnaseq.github.io/) without digital normalization according to the parameters of min_kmer_cov $=2$ and other default parameters $[26,27]$. Short reads with overlap regions were assembled into longer contigs. Then contigs were linked into transcripts (the length less than 200 bp were filtered) and the transcripts clustered based on nucleotide sequence identity. The longest transcripts in the cluster units were regarded as unigenes to eliminate redundant sequences. These unigenes were annotated using the BLASTx alignment $\left(E\right.$-value $\left.\leq 10^{-5}\right)$ to various protein databases, including the NCBI non-redundant (NR) protein database (ftp://ftp.ncbi.nih.gov/blast/db/), the Swiss-Prot protein database (http://www.uniprot.org/), the Kyoto Encyclopedia of Genes and Genomes (KEGG) pathway database (http://www.genome.jp/ $\mathrm{kegg} /$ ), the Clusters of Orthologous Groups of proteins (COG) database (http://www.ncbi.nlm.nih.gov/ COG/), the eukaryotic Orthologous Groups of proteins (KOG) database (http://www.ncbi.nlm.nih.gov/ COG/), and the Gene Ontology (GO) database (http://www.geneontology.org/). After predicting the amino acid sequences of unigenes by GetOrf (EMBOSS:v6.3.1), we aligned these sequences in Protein family (Pfam, http://pfam.xfam.org/) using HMMER software suite (v3.0) $\left(E\right.$-value $\left.\leq 10^{-10}\right)$. The best alignment results from seven databases were chosen to determine the annotation of the unigenes.

\section{Expression annotation}

The alignment package Bowtie (v4.4.7) was used to map reads back to unigenes. According to the comparison results, the expression levels were estimated by means of RSEM (RNA-Seq by expectation maximization) [28, 29]. Unigene expression abundance differences among the samples were represented using Fragments Per Kilobase 
of transcript per Million mapped reads (FPKM) method [30]. Different expression analysis among different stages was performed with DESeq package (v1.18.0) [31]. Differentially expressed genes (DEGs) were determined based on false discovery rate $(\mathrm{FDR}<0.01)$ and fold change $(\mathrm{FC} \geq 2)$.

\section{GO and KEGG pathway enrichment analyses for differentially expressed unigenes}

GO and KEGG pathway enrichment analyses for the differentially expressed unigenes were then carried out. The obtained GO annotation was enriched and refined using topGo package (v2.12.0) with the "elim" method and Kolmogorov-Smirnov test. KEGG pathways were enriched using in-house scripts according to Fisher's exact test. Enriched $p$-values were calculated and adjusted with the Bonferroni correction. We selected the corrected $p$-value of 0.05 as the threshold to determine significant enrichment of the gene sets.

\section{Gene validation and expression analysis}

For the purpose of gene validation and expression analysis, all the DEGs related to flavonoid biosynthesis were subjected to real-time quantitative PCR (RT-qPCR) on ABI StepOnePlus ${ }^{\text {Tix }}$ Real-Time PCR System (Applied Biosystems, USA). Primer sequences designed with Primer Premier 5.0 software are shown in Additional file 1: Table S1. cDNA synthesis and RT-qPCR were performed using FastQuant RT Kit (With gDNase) and SuperReal PreMix Plus (SYBR Green) (TIANGEN Biotech (Beijing) Co., Ltd., China). Actin 11 was selected as an internal control [32]. At least three independent biological replicates for each sample and three technical replicates of each biological replicate were arranged to ensure the reproducibility and reliability of RT-qPCR results.

\section{Results}

Contents of flavonoids in the petals of Nymphaea 'King of Siam'

To understand the color formations in Nymphaea 'King of Siam, we compared the metabolic profiles of petals. As expected, anthocyanins were not detected in S1, while four anthocyanins, namely delphinidin $3-O-\beta-$ galactopyranoside (a1, Dp3Gal), delphinidin 3'-O-(2"-Ogalloyl- $\beta$-galactopyranoside (a2, Dp3' galloylGal), delphinidin 3-O-(6"-O-acetyl- $\beta$-glucopyranoside (a3, Dp3acetylGlc), delphinidin 3'-O-(2" -O-galloyl-6" -O-acetyl- $\beta$ galactopyranoside (a4, Dp3'galloyl-acetylGal) were detected in other stages. Furthermore, we compared the intermediate products involved in flavonoid biosynthesis. A total of 12 kinds of flavonols, including myricetin derivatives, quercetin derivatives, and kaempferol derivatives were detected in petals. In addition, myricetin 3-O-
$\alpha$-L-(3"'-O-malonyl)-rhamnopyranoside, myricetin 3-O(2"-O-galloyl-6" -O-malonyl- $\beta$-galactopyranoside), quercetin 3-O- $\alpha-\mathrm{L}-(3$ "'-O-malonyl)-rhamnopyranoside, and kaempferol 3-O- $\alpha-\mathrm{L}-\left(3^{\prime \prime}-O\right.$-malonyl)-rhamnopyranoside were first detected in waterlily. The retention time, ultraviolet-visible spectral properties and mass spectrometric data, and content of each compound are listed in Additional file 2: Table S2.

These results showed that the content of each composition varied with the coloring stage (Fig. 2). The total anthocyanins content (TA) ranged from 0 to $83.16 \mathrm{mg}$ $100 \mathrm{~g}^{-1} \mathrm{FW}$. Dp3'G (including a2 and a4) were the domain anthocyanins and the highest content of Dp3'G (74.26 mg $100 \mathrm{~g}^{-1} \mathrm{FW}$ ) was detected in S4 (Fig. 2a). The total flavonols content (TF) were about 16.41$585.53 \mathrm{mg} 100 \mathrm{~g}^{-1} \mathrm{FW}$ and TF in S5 was nearly five times higher than that in S1 (Fig. 2b). Myricetin derivatives were the main components of flavonols and accounted for approximately 39.01 to $88.67 \%$ of TF, followed by quercetin derivatives $(9.84 \%$ of TF in S5 and $61.00 \%$ of TF in S1). The contents of kaempferol derivatives were lower in petals and only detected in S4, S5, and S6 (Additional file 3: Table S3).

\section{RNA-Seq and de novo assembly}

To deeply understand the molecular mechanism of blue color formation in waterlily petals, six libraries (S1-1, S1-2, S1-3, S3-1, S3-2, and S3-3) were constructed with blue waterlily cultivar Nymphaea 'King of Siam' in two different coloring stages and each stage was repeated three times. S1-1, S1-2, and S1-3 were the libraries of the first stage when the petals were colorless; S3-1, S3-2, and S3-3 were the third stage when the outer petals were fully colorated and inner petals were colorless (S1 and S3 in Fig. 1). The six libraries were sequenced by using an Illumina HiSeq $^{\text {Th }} 2500$ platform with 125 paired-end reads (NCBI accession: SRP081259). After cleaning and quality checking, about $36.99 \mathrm{~Gb}$ total clean reads were obtained, and each library produced no less than $5.65 \mathrm{~Gb}$ clean reads. Q30 percentage (percentage of bases with sequencing error rate lower than $1 \%$ ) were $90.87 \%, 90.93 \%, 90.55 \%, 90.90 \%$, $90.78 \%$, and $90.84 \%$, respectively. These data showed that the RNA-Seq quality was applicable for further analysis (Additional file 4: Table S4).

Subsequently, de novo assembly generated 329,005 transcripts and 112,485 unigenes, with the N50 (covering $50 \%$ of all the nucleotide sequences of the largest unigene length) of 2,418 nt and 1,496 nt. In total, 63,097 unigenes (56.09\%) were between 200 and 500 nt; 38,064 unigenes (33.84\%) were between 500 and 1000 nt; 11,324 unigenes $(10.07 \%)$ were longer than $2000 \mathrm{nt}$ (Table 1). 

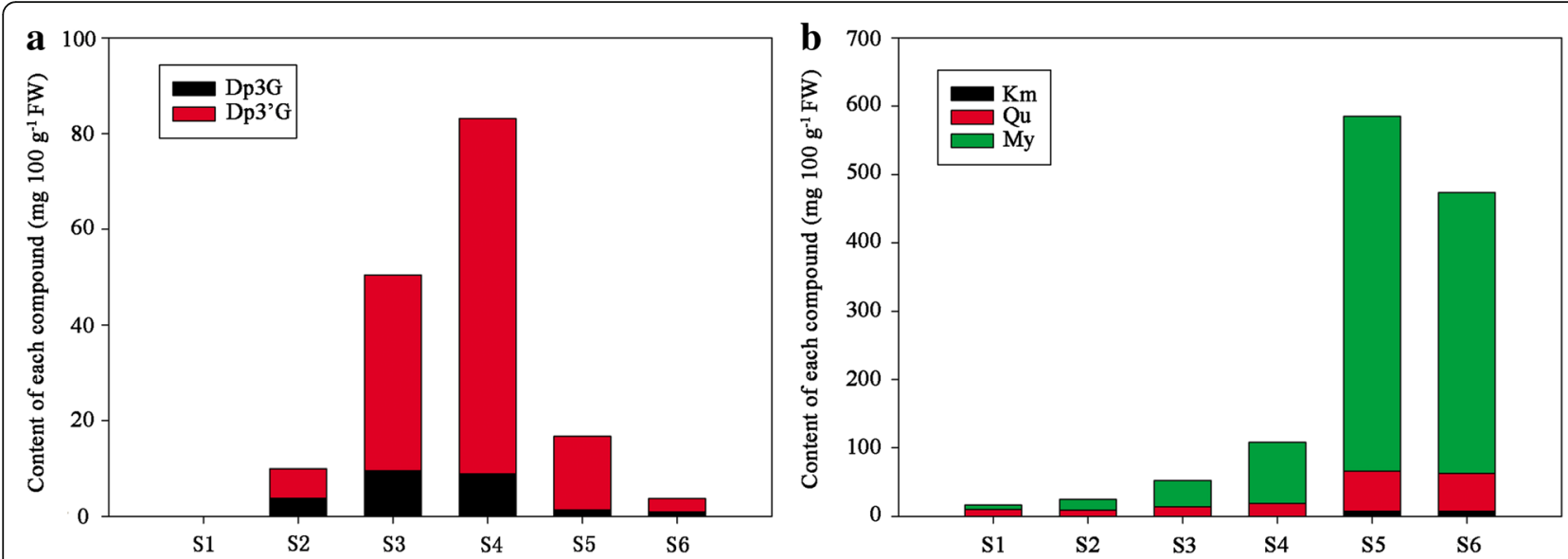

Fig. 2 Flavonoid composition obtained by UPLC from six different coloring stages (S1, S2, S3, S4, S5, and S6). Fig. 2a showed the content of anthocyanins. Dp3G contain the content of delphinidin 3-O- $\beta$-galactopyranoside (Dp3Gal) and delphinidin 3-O-(6"-O-acetyl- $\beta$-glucopyranoside) (Dp3acetylGIC); Dp3'G contain the content of delphinidin 3'-O-(2"-O-galloyl- $\beta$-galactopyranoside) (Dp3'galloylGal) and delphinidin 3'-O-(2"-Ogalloyl-6"-O-acetyl- $\beta$-galactopyranoside) (Dp3'galloyl-acetylGal). Fig. $2 \mathrm{~b}$ indicated the content of flavonols. Km contain the content of kaempferol 3-(3"-acetylrhamnoside) and kaempferol 3-O-a-L-(3"-O-malonyl)-rhamnopyranoside. Qu contain the content of quercetin 7-O-galactoside, quercetin 3-O-a-L-rhamnopyranoside, quercetin 3-O-B-D-(3"-O-acetyl)-a-L-rhamnopyranoside and quercetin the content of 3-O-a-L-(3"-O-malonyl)rhamnopyranoside. My contain the content of myricetin 3-O- $\beta$-D-galactopyranoside, myricetin 3-O-a-L-rhamnopyranoside, myricetin 3-O-a-L-(3"O-acetyl)-rhamnopyranoside, myricetin 3-O-a-L-(2"-O-acetyl)-rhamnopyranoside, myricetin 3-O-a-L-(3"-O-malonyl)-rhamnopyranoside and myricetin 3-O-(2"-O-galloyl-6"-O-malonyl- $\beta$-galactopyranoside)

\section{Functional annotation}

Due to the lack of a complete genome sequence in Nymphaea, only 26,206 unigenes were annotated to seven databases (NR, Swiss-Prot, KOG, COG, GO, KEGG, and Pfam), which accounted for about $23.3 \%$ of 112,485 unigenes. Among them, 25,720 unigenes (98.15\% of all annotated unigenes) could be annotated to the NCBI NR database, while 17,627 (67.26\%), 16,331 (62.32\%) and 15,308 (58.41 \%) unigenes could be annotated to SwissProt, Pfam and KOG databases. We annotated 13,526 (51.61 \%), 7,968 (30.41\%) and 5,209 (14.39\%) unigenes to GO, COG and KEGG databases. According to the NCBI NR annotation and $E$-value distribution, $53.03 \%$ of the annotated sequences had strong homology ( $E$ value $<10^{-50}$ ) and $33.75 \%$ of the annotated sequences

Table 1 Length distribution of contigs, transcripts, and unigenes in Nymphaea 'King of Siam' petals transcriptome

\begin{tabular}{llll}
\hline Length Range & Contigs & Transcripts & Unigenes \\
\hline $200-300$ & $19,842,599(99.50 \%)$ & $50,671(15.40 \%)$ & $36,424(32.38 \%)$ \\
$300-500$ & $37,313(0.19 \%)$ & $45,986(13.98 \%)$ & $26,673(23.71 \%)$ \\
$500-1000$ & $33,056(0.17 \%)$ & $60,487(18.38 \%)$ & $23,243(20.66 \%)$ \\
$1000-2000$ & $20,027(0.10 \%)$ & $80,138(24.36 \%)$ & $14,821(13.18 \%)$ \\
2000+ & $10,181(0.05 \%)$ & $91,723(27.88 \%)$ & $11,324(10.07 \%)$ \\
Total number & $19,943,175$ & 329,005 & 112,485 \\
Total length & $845,487,394$ & $490,152,433$ & $94,130,265$ \\
N50 length & 43 & 2,418 & 1,496 \\
Mean length & 42.39 & 1489.8 & 836.83 \\
\hline
\end{tabular}

showed very strong homology $\left(E\right.$-value $<10^{-100}$ ) (Fig. 3a). The 25 top-hit species based on NCBI NR annotation are shown in Fig. 3b. Nearly $50 \%$ of unigenes could be annotated with the sequences from the 5 top-hit species, such as Nelumbo nucifera, Amborella trichopoda, Vitis vinifera, Elaeis guineensis, and Phoenix dactylifera, while we found that more than $19 \%$ of the sequences showed the highest homology to the sequences from Nelumbo nucifera.

17,627 unigenes were annotated against the SwissProt database, accounting for $67.26 \%$ of all annotated unigenes and 121 unigenes that were not annotated against the other three databases. Pfam is the most comprehensive domain annotation of protein structure classification system. In this study, there were 16,331 unigenes noted to Pfam database.

KOG analysis was used to searching orthologous classification and gene evolution rates. There were 15,308 unigenes ( $58.41 \%$ of all annotated unigenes) that aligned with 25 KOG classifications. Out of the 25 KOG categories, the cluster for general function prediction only (31.86 \%) represented the largest group, followed by posttranslational modification, protein turnover, chaperones $(8.16 \%)$ and signal transduction mechanisms (7.11 \%). Only a small proportion (lower than $1 \%$ ) of unigenes was assigned to the number of unigenes which involved in nuclear structure, cell motility and extracellular structure. It is worth noting that a large number of unigenes were assigned to carbohydrate transport and metabolism (4.75\%), translation, ribosomal structure and biogenesis (4.34\%), 


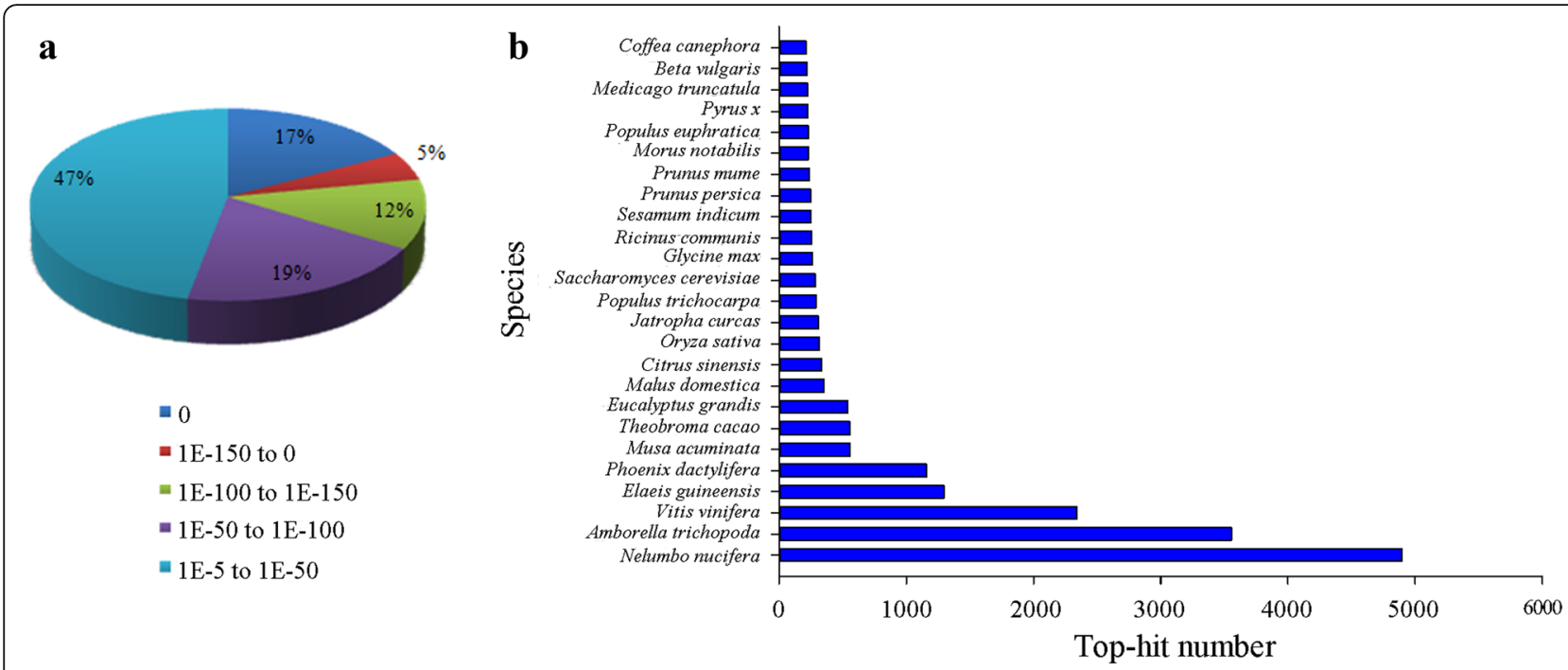

Fig. 3 Characteristics of homology search of Nymphaea 'King of Siam' unigenes. (a) E-value distribution of the top BLASTx hits against the NR database for each unigene. (b) Number and percentage of unigenes matching the top 25 species using BLASTx in the NR database

transcription (4.33\%), energy production and conversion (3.92\%), intracellular trafficking, secretion, and vesicular transport (3.80 \%) and secondary metabolites biosynthesis, transport and catabolism (3.43 \%) (Fig. 4).

\section{Identification and GO functional enrichment analysis of DEGs} A total of 1,581 DEGs were obtained comparing S1 and S3 (Additional file 5: Figure S1). Among these, 558 DEGs were assigned one or more GO terms. These DEGs provided clues about the molecular events related to petal development especially petal color development. TopGO package (v2.12.0) was used to map all DEGs in the GO database to exploring the significantly enriched terms compared with the genome backgraound, and corrected $p$-value of 0.05 (Fisher's exact test) was the threshold. There were a total of 10 enrichment GO terms among three gene ontology categories (Table 2). The results showed that oxidation-reduction process, lipid biosynthetic process, urea transmembrane transport, anthocyanin-containing compound biosynthetic process, pattern specification process and response to light stimulus were the most significant enrichment GO terms under the biological process category. Urea transmembrane transporter activity, peroxidase activity,

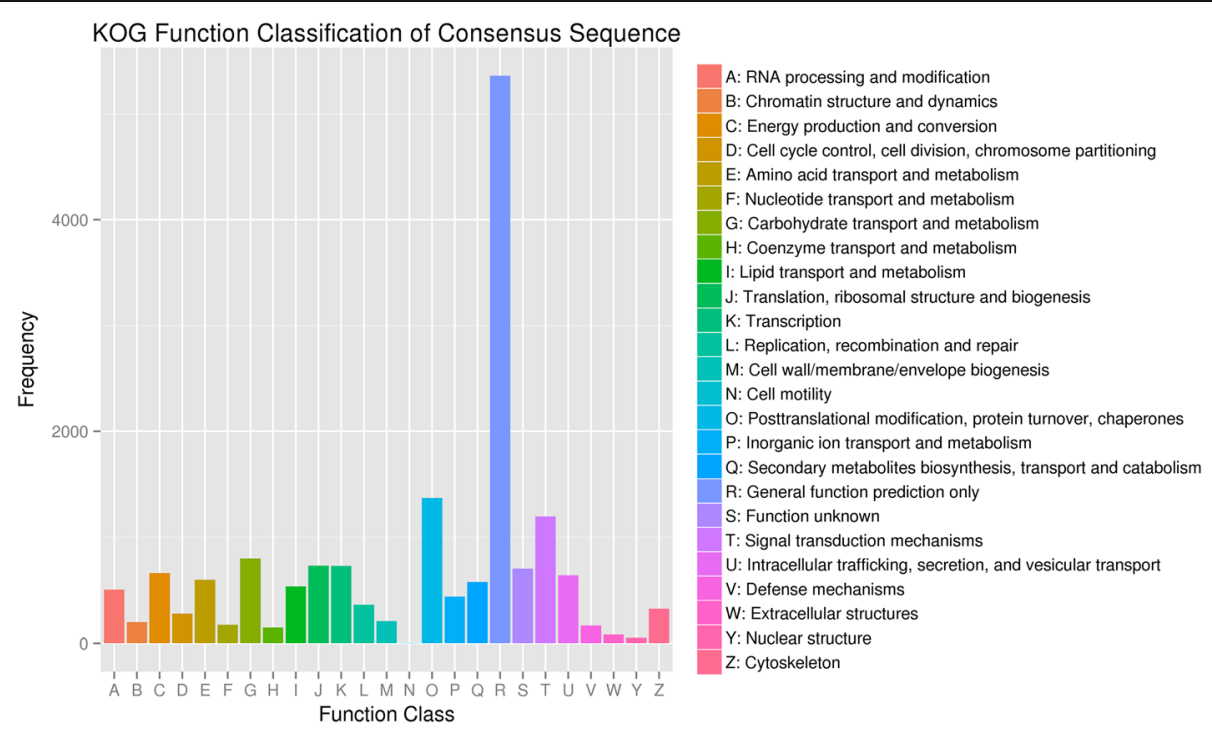

Fig. $4 \mathrm{KOG}$ categories of the annotated unigenes 
Table 2 Gene Ontology functional enrichment analysis of DEGs in Nymphaea 'King of Siam' petals transcriptome

\begin{tabular}{|c|c|c|c|c|}
\hline Gene Ontology term & Cluter frequency & Genome frequency & $P$-value & $\begin{array}{l}\text { Corrected } \\
p \text {-value }\end{array}$ \\
\hline Biological Process: oxidation-reduction process (GO:0055114) & $\begin{array}{l}59 \text { out of } 406 \\
14.5320197044335 \%\end{array}$ & $\begin{array}{l}773 \text { out of } 9953 \\
7.7665025620416 \%\end{array}$ & $1.65 \mathrm{E}-06$ & 0.000753462 \\
\hline Biological Process: lipid biosynthetic process (GO:0008610) & $\begin{array}{l}6 \text { out of } 406 \\
1.47783251231527 \%\end{array}$ & $\begin{array}{l}15 \text { out of } 9953 \\
0.150708329146991 \%\end{array}$ & $1.62 \mathrm{E}-05$ & 0.007422674 \\
\hline Biological Process: urea transmembrane transport (GO:0071918) & $\begin{array}{l}4 \text { out of } 406 \\
0.985221674876847 \%\end{array}$ & $\begin{array}{l}6 \text { out of } 9953 \\
0.0602833316587963 \%\end{array}$ & 3.83E-05 & 0.017522614 \\
\hline $\begin{array}{l}\text { Biological Process: anthocyanin-containing compound biosynthetic } \\
\text { process (GO:0009718) }\end{array}$ & $\begin{array}{l}5 \text { out of } 406 \\
1.23152709359606 \%\end{array}$ & $\begin{array}{l}11 \text { out of } 9953 \\
0.11051944137446 \%\end{array}$ & 4.15E-05 & 0.018979757 \\
\hline Biological Process: pattern specification process (GO:0007389) & $\begin{array}{l}7 \text { out of } 406 \\
1.72413793103448 \%\end{array}$ & $\begin{array}{l}26 \text { out of } 9953 \\
0.261227770521451 \%\end{array}$ & $5.98 \mathrm{E}-05$ & 0.027344686 \\
\hline Biological Process: response to light stimulus (GO:0009416) & $\begin{array}{l}10 \text { out of } 406 \\
2.46305418719212 \%\end{array}$ & $\begin{array}{l}56 \text { out of } 9953 \\
0.562644428815433 \%\end{array}$ & 7.55E-05 & 0.034521692 \\
\hline $\begin{array}{l}\text { Molecular Function: urea transmembrane transporter activity } \\
\text { (GO:0015204) }\end{array}$ & $\begin{array}{l}4 \text { out of } 437 \\
0.91533180778032 \%\end{array}$ & $\begin{array}{l}6 \text { out of } 10874 \\
0.0551774875850653 \%\end{array}$ & $3.62 \mathrm{E}-05$ & 0.009120301 \\
\hline Molecular Function: peroxidase activity (GO:0004601) & $\begin{array}{l}11 \text { out of } 437 \\
2.51716247139588 \%\end{array}$ & $\begin{array}{l}69 \text { out of } 10874 \\
0.634541107228251 \%\end{array}$ & 8.64E-05 & 0.021766185 \\
\hline $\begin{array}{l}\text { Molecular Function: sequence-specific DNA binding transcription } \\
\text { factor activity (GO:0003700) }\end{array}$ & $\begin{array}{l}17 \text { out of } 437 \\
3.89016018306636 \%\end{array}$ & $\begin{array}{l}155 \text { out of } 10874 \\
1.42541842928085 \%\end{array}$ & 1.60E-04 & 0.040431117 \\
\hline Molecular Function: heme binding (GO:0020037) & $\begin{array}{l}18 \text { out of } 437 \\
4.11899313501144 \%\end{array}$ & $\begin{array}{l}170 \text { out of } 10874 \\
1.56336214824352 \%\end{array}$ & $1.63 \mathrm{E}-04$ & 0.041003922 \\
\hline
\end{tabular}

sequence-specific DNA binding transcription factor activity and heme binding were the most enriched in the molecular function category.

\section{KEGG functional enrichment analysis of DEGs}

Pathway analysis is helpful to understand the biological functions and gene interactions. There were 134 DEGs mapped into 63 enriched pathways which contained five main categories while only 4 pathways were selected ( $q$ value < 0.05) (Additional file 6: Table S5). The most represented pathways included phenylalanine metabolism (ko00360, 20 DEGs), phenylpropanoid biosynthesis (ko00940, 24 DEGs), followed by flavonoid biosynthesis (ko00941, 21 DEGs) and stilbenoid, diarylheptanoid and gingerol biosynthesis (ko00945, 10 DEGs). From these pathways, information concerning Nymphaea 'King of Siam' metabolism in two different coloring stages can be obtained. Then, we could understand metabolic pathways more clearly.

\section{Genes related to color development}

We analyzed the unigenes involved in flavonoid biosynthesis, anthocyanin biosynthesis, and flavone and flavonol biosynthesis pathways in Nymphaea 'King of Siam' which were related to flower pigmentation. We searched all these genes in the combined functional annotations. A total of 127 unigenes involved in the above three pathways were the study focus (Table 3 ). Then, we analyzed transcriptional profiles of the genes involved in flavonoid metabolism especially anthocyanin biosynthetic pathway between S1 and S3 to find the key transcripts in the metabolism of blue color. The results demonstrated that a total of 33 key unigenes had significant changes in expression levels, including 28 up-regulated and 5 downregulated unigenes (Additional file 1: Table S1). All these DEGs contained both upstream genes ( $\mathrm{CHS}, \mathrm{CHI}$, etc.) and downstream genes (UA3GT, etc.) (Fig. 5). Among them, more than one unigene was annotated as the same enzyme. The formation of blue pigment in waterlily mainly relied on hydroxylation, glycosylation, and acylation rather than methoxylation to maintain its stability, because both the sequences for methoxylation genes involved in anthocyanin modification and the methylated anthocyanin were absent.

In order to verify the credibility of transcriptome sequencing data, the sequences of all these 33 core unigenes, which were not only associated with waterlily color formation but also had different expression levels, were subjected to fluorescent quantitative analysis of RT-qPCR with the designed primers. The results showed that transcriptome sequencing data were credible because the correlation coefficient between transcriptome sequence and RT-qPCR was high $\left(\mathrm{R}^{2}=0.6634\right)$ (Additional file 7: Figure S2). Our results of RT-qPCR were almost consistent with those obtained with RNA-Seq method. As shown in Fig. 6, RT-qPCR was also used to study the expression patterns of seven structural unigenes $\left(F 3^{\prime} H, F 3^{\prime} 5^{\prime} H, \quad D F R, F L S\right.$, $U A 3 G T-4, U A 3 G T-5$, and UA3GT-6) in six different coloring stages. These seven unigenes had similar expression patterns and their expression curves were "bell curves". With the development of petals, their 
Table 3 Candidate genes related to flower pigmentation of Nymphaea 'King of Siam'

\begin{tabular}{|c|c|c|c|c|c|c|}
\hline Function & Gene & Enzyme & ko id (EC no.) & No.All & $\begin{array}{l}\text { No. } \\
\text { Up }\end{array}$ & $\begin{array}{l}\text { No. } \\
\text { Down }\end{array}$ \\
\hline \multirow[t]{7}{*}{ Anthocyanin biosynthesis } & $\mathrm{CHS}$ & Chalcone synthase & k00660 (2.3.1.74) & 10 & 2 & 0 \\
\hline & $\mathrm{CHI}$ & Chalcone isomerase & k01859 (5.5.1.6) & 2 & 1 & 0 \\
\hline & $\mathrm{F} 3 \mathrm{H}$ & Flavanone 3-hydroxylase & k00475 (1.14.11.9) & 8 & 5 & 0 \\
\hline & $F 3^{\prime} H$ & Flavonoid 3'-hydroxylase & $\begin{array}{l}\mathrm{k} 05280 \\
(1.14 .13 .21)\end{array}$ & 11 & 1 & 0 \\
\hline & $F 3^{\prime} 5^{\prime} H$ & Flavonoid 3',5'-hydroxylase & $\begin{array}{l}\mathrm{k} 13083 \\
(1.14 .13 .88)\end{array}$ & 8 & 1 & 0 \\
\hline & $D F R$ & Dihydroflavonol 4-reductase & k13082 (1.1.1.219) & 5 & 1 & 0 \\
\hline & ANS & Anthocyanidin synthase & $\begin{array}{l}\mathrm{k} 05277 \\
(1.14 .11 .19)\end{array}$ & 3 & 3 & 0 \\
\hline \multirow[t]{8}{*}{ Anthocyanin modificatioon } & UA3GT & Anthocyanidin 3-O-glucosyltransferase & k12903 (2.4.1.115) & 27 & 3 & 3 \\
\hline & UGT75C1 & Anthocyanidin 3-O-glucoside 5-O-glucosyltransferase & k12338 (2.4.1.298) & 4 & 1 & 0 \\
\hline & GT1 & Anthocyanidin 5, 3-O-glucosyltransferase & k12938 (2.4.1.-) & 7 & 1 & 2 \\
\hline & $3 G G T$ & Anthocyanidin 3-O-glucoside 2"-O-glucosyltransferase & k12933 (2.4.1.297) & 3 & 0 & 0 \\
\hline & $3^{\prime} G T$ & Anthocyanidin 3'-O-glucosyltransferase & k12939 (2.4.1.238) & 6 & 0 & 0 \\
\hline & $A A 7 G T$ & $\begin{array}{l}\text { Cyanidin 3-O-glucoside 5-O-glucosyltransferase } \\
\text { (acyl-glucose) }\end{array}$ & k17194 (2.4.1.299) & 1 & 0 & 0 \\
\hline & AA5GT & $\begin{array}{l}\text { Cyanidin 3-O-glucoside 7-O-glucosyltransferase } \\
\text { (acyl-glucose) }\end{array}$ & k17192 (2.4.1.300) & 1 & 0 & 0 \\
\hline & $5 A T$ & Anthocyanidin 5-O-aromatic acyltransferase & k12936 (2.3.1.153) & 1 & 1 & 0 \\
\hline \multirow[t]{7}{*}{$\begin{array}{l}\text { Flavone and flavonol } \\
\text { biosynthesis }\end{array}$} & FNS & Flavone synthase & $\begin{array}{l}\mathrm{k} 13077 \\
(1.14 .11 .22)\end{array}$ & 1 & 0 & 0 \\
\hline & $F L S$ & Flavonol synthase & $\begin{array}{l}\mathrm{k} 13078 \\
(1.14 .11 .23)\end{array}$ & 6 & 1 & 0 \\
\hline & C12RT1 & $\begin{array}{l}\text { Flavanone 7-O-glucoside 2"-O-beta-L- } \\
\text { rhamnosyltransferase }\end{array}$ & k13080 (2.4.1.236) & 2 & 0 & 0 \\
\hline & GUSB & beta-Glucuronidase & k01192 (3.2.1.31) & 1 & 0 & 0 \\
\hline & UF3GT & Flavonol 3-O-glucosyltransferase & k10757 (2.4.1.91) & 11 & 4 & 0 \\
\hline & FOMT & Flavonol 3-O-methyltransferase & k05279 (2.1.1.76) & 5 & 2 & 0 \\
\hline & CROMT2 & Myricetin O-methyltransferase & k13272 (2.1.1.149) & 1 & 0 & 0 \\
\hline Flavanone biosynthesis & ANR & Anthocyanidin reductase & k08695 (1.3.1.77) & 3 & 1 & 0 \\
\hline
\end{tabular}

expression levels increased gradually, reached the maximum level in S4, and then decreased in S5 and S6. FLS and UA3GT-6 had the most rapid change in different stages. In Nymphaea 'King of Siam', changes in expression of genes encoding pathway enzymes have corresponded to changes in anthocyanin accumulation like many other cases [22, 23, 33, 34].

\section{Discussion}

Waterlily is not only an important ornamental plant in the water, but also have many medicinal effects [35, 36]. Tropical waterlily flowers have the special colors of blue, violet, and bluish purple, while hardy waterlily flowers lack of the above colors. However, the distribution of tropical waterlily is limited because it can only grow in a warm winter, while hardy waterlily is applicable to various grow conditions and requires less labor. For these reasons, breeding blue hardy waterlily can not only satisfy the demand of novel color, but also save cultivation costs. To breed blue hardy waterlily, firstly we should understand the formation mechanism of blue color in tropical waterlily.

Due to the lack of a reference genome sequences, it is difficult to understand the molecular mechanism of blue color formation in tropical waterlily. In the study, we sequenced and comparatively analyzed the transcriptome of the different coloring stages of blue waterlily cultivar Nymphaea 'King of Siam' petals with the next-generation sequencing technology. A total of 16 flavonoids, including 4 anthocyanins and 12 flavonols were detected in petals of Nymphaea 'King of Siam'. The detected flavonols contained three aglycones, such as myricetin, quercetin, and kaempferol, and the contents of myricetin derivatives were higher. In addition, only delphinidin (Dp) derivatives, such 


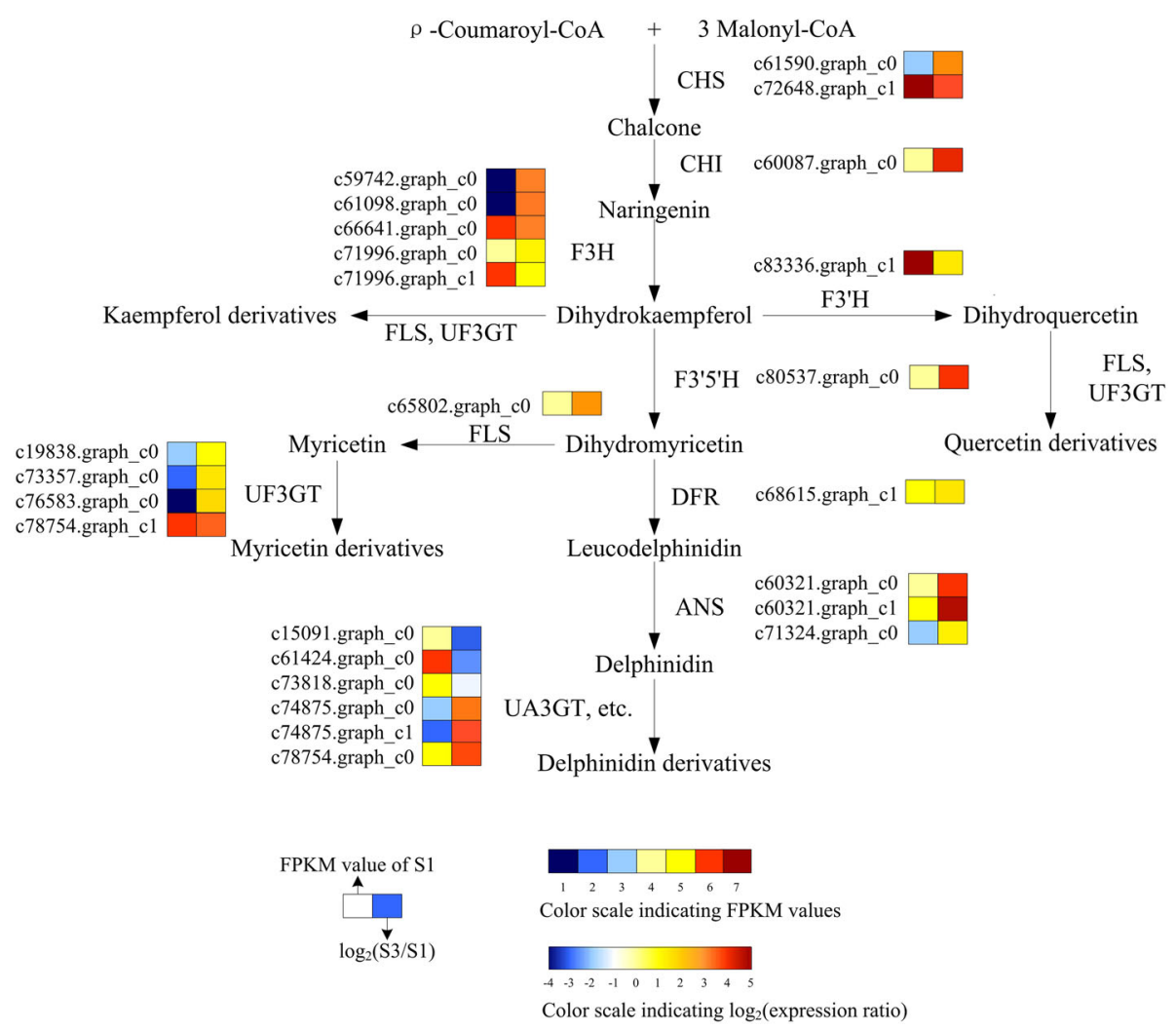

Fig. 5 A detailed part of flavonoid metabolic subnetwork showing the subset of nodes or metabolites that constitute the process. Enzyme names, unigene names and expression patterns are indicated at the side of each step. The expression pattern of each unigene is shown on two grids, with the left one representing the FPKM value of $\mathrm{S} 1$, and the right one representing the relative $\log _{2}(\mathrm{~S} 3 / \mathrm{S} 1)$. The grids with seven different colors show the absolute expression magnitude of S1, withV the FPKM values $0-1,1-2,2-4,4-8,8-16,16-32$, and 32-64 represented by different scale levels 1-7, respectively. CHS: chalcone synthase; CHI: chalcone isomerase; F3H: flavanone 3-hydroxylase; F3'H: flavonoid 3'-hydroxylase; F3'5' H: flavonoid 3'5'-hydroxylase; FLS: flavonol synthase; UF3GT: flavonol 3-O-glucosyltransferase; DFR: dihydroflavonol 4-reductase; ANS: anthocyanidin synthase; UA3GT: anthocyanidin 3-O-glucosyltransferase

as Dp3Gal, Dp3'galloylGal, Dp3acetylGlc, and Dp3'galloyl-acetylGal were detected. Therefore, we focused on the biosynthesis of Dp derivatives. Among all unigenes involved in the Dp biosynthesis process, two $C H S$, one $C H I$, five $F 3 H$, one $F 3^{\prime} H$, one $F 3^{\prime} 5^{\prime} H$, one $D F R$, three $A N S$, and three $U A 3 G T$ homologous sequences showed upregulated expression levels in S3 compared to those in S1. These unigenes covered almost the complete Dp biosynthetic pathway. Blue flower formation was comprehensively regulated by multiple unigenes.

Hydroxylation of flavanones to dihydroflavonols are mediated by $\mathrm{F} 3 \mathrm{H}, \mathrm{F} 3{ }^{\prime} \mathrm{H}$, or $\mathrm{F}^{\prime} 5^{\prime} \mathrm{H}$. $F 3^{\prime} H$ and $F 3^{\prime} 5^{\prime} H$ are called "red gene" and "blue gene" because they are required for $\mathrm{Cy}$ and $\mathrm{Dp}$ production, respectively. $\mathrm{F}^{\prime} \mathrm{H}$ and $\mathrm{F}^{\prime}{ }^{\prime}{ }^{\prime} \mathrm{H}$ are the key enzymes determining the structures of anthocyanins and the color [37]. Many important floricultural crops, such as Carnations, Tulipa, Rosa hybrida, and Chrysanthemum morifolium, do not produce Dp and thus lack violet/blue color cultivars. This is attributed to the fact that these crops do not possess the F3'5' $H$ gene. Transgenic violet/blue carnations [38], roses [39], and chrysanthemums [40, 41] were developed by expressing a heterologous $F 3^{\prime} 5^{\prime} H$ gene. Eight $F 3^{\prime} 5^{\prime} H$ were found in Nymphaea 'King of Siam', whereby one $F 3^{\prime} 5^{\prime} H$ expression was up-regulated in $\mathrm{S} 3\left(\log _{2} \mathrm{FC}=4.17\right)$. As showed in Fig. 5, $F^{\prime} 5^{\prime} \mathrm{H}$ was crucial in myricetin derivatives and $\mathrm{Dp}$ derivatives synthesis. $F 3^{\prime} 5^{\prime} H$ expression levels increased from $\mathrm{S1}$, then reached the maximum level in S4, decreased in S5 and S6 (Fig. 6), and the highest total content of myricetin derivatives and $\mathrm{Dp}$ derivatives were approximately $535.9 \mathrm{mg} 100 \mathrm{~g}^{-1} \mathrm{FW}$ in S5 (Additional file 3: Table S3). All these analysis indicated that the change of expression level corresponded to the changes in the content of myricetin derivatives and $\mathrm{Dp}$ derivatives.

DFR provides one entry step to the biosynthesis of anthocyanins, and can utilized any one or three of the dihydroflavonols (dihydromyricetin, dihydrokaempferol, hyhydroquercetin), resulting in the formation of corresponding leucoanthocyanidins. The three substrates of DFR are similar in the structure and only different in the number of hydroxyl groups on the B phenyl ring, which 

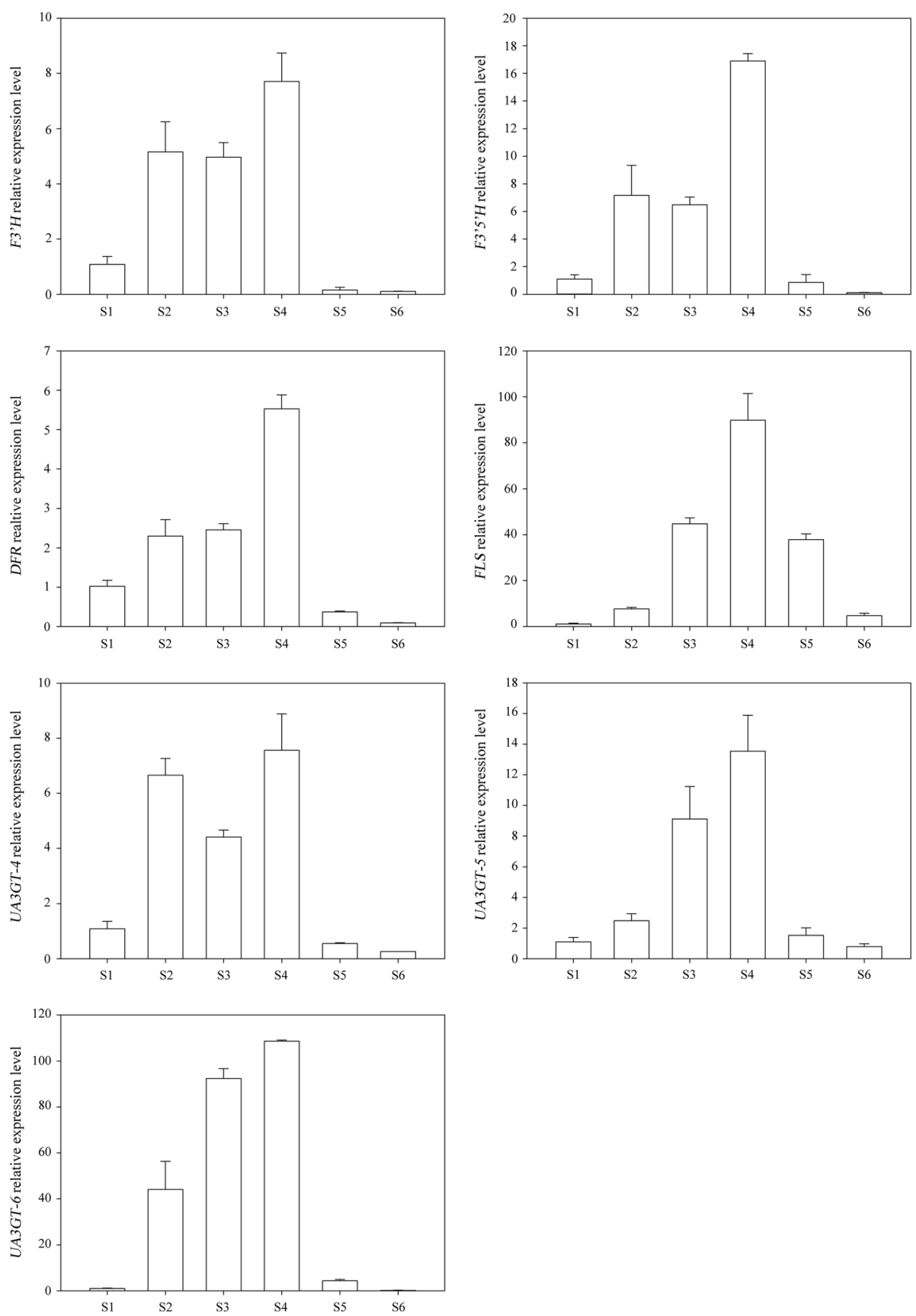

Fig. 6 Expression patterns of seven candidate unigenes from six different coloring stages. Actin 11 was used as an internal control. Each unigenes has three biological replicates, and each biological replicate has three technical replicates

is not the site of enzymatic action. It is not surprising that DFRs from many plant species can utilize all three dihydroflavonols. However, in some species, DFR has strict substrate specificity [42]. In this study, pelargonidin or cyanidin-based anthocyanins have not been detected. There are two possible explanations for this. Firstly, DFRs in Nymphaea 'King of Siam' only utilize dihydromyricetin. Secondly, DFRs utilize dihydromyricetin, dihydroquercetin, and dihydrokaempferol, but FLS can utilize dihydroquercetin and dihydrokaempferolin priority. The competitiveness of $D F R / F L S$ was significant to Nymphaea 'King of Siam' because it could change both content of flavonols and anthocyanins. In this study, expression level of FLS was higher than DFR. Therefore, the contents of flavonols were higher than anthocyanins. As co-pigment, flavonols played an important role in 
color formation. Change the expression level of DFR/ FLS may lead to varying degrees to change the color in waterlily.

Anthocyanins are modified at one or several positions by methylation, glycosylation, or acylation. These modifications endow anthocyanins with unique properties, including color shade, stability, and solubility [43]. Methylation is not as important as glycosylation or acylation, because methylated anthocyanin were not detected in the petals of Nymphaea 'King of Siam, and the sequences of methoxylation genes involved in anthocyanin modification were not obtained in assembled results. Metabolites of anthocyanins had been compared in previous studies [25, 44-47]. In tropical waterlily, delphindin 3'glycosides were the main anthocyanins in blue cultivars; delphindin 3'-glycosides and delphindin 3-glycosides were detected in blue/violet ones; only delphindin 3-glycosides were found in violet/red petals. In red cultivars, cyanidin 3 -glycosides and delphindin 3-glycosides were detected both in red tropical and hardy waterlily. The determined contents of anthocyanins in white, yellow, and pink cultivars were low or zero, indicating that the structural modification (especially 3'-position modification) by down-stream genes was crucial to blue color formation. Therefore, 3'-glycosyltransferases (3'GTs) might play an important role in blue petal color formation of waterlily. 3'GTs provide the basis of 3'-polyacylations. Two 3'GT have been identified: GtUA3'GlcT of Gentiana triflora and anthocyanin 3', 5'-glucosyltransferase (CtUA3'5' GlcT) of Clitoria ternatea [7, 48, 49].

Six $U A 3^{\prime} G T$ homologous unigenes were found in waterlily (Additional file 8: Table S6). However, their FPKM were very low, expression levels had no significant change between S1 and S3. For this reason, these six UA3'GT may be not the key enzymes for blue formation in waterlily. Different from other species, waterlily may have other unigenes which can also catalyse 3 'position glycosides. Kogawa et al. [49] found that UA3' 5'GT was important in Clitoria ternatea which accumulated polyacylated anthocyanins named ternatins, UA3' 5 'GT could be glycoslated at $3{ }^{\prime}$-, 5' -position of delphindin. Ogata et al. [50] had reported that RhGT1 could be glycosylated at two different sites (3-, 5-position) of cyanidin in roses. In this study, we compared the expression levels and patterns of all glycosyltransferase in anthocyanin biosynthetic pathway and found that UA3GTs might be the most important enzymes for the accumulation of delphindin 3'-glycosides which are the main anthocyanins in blue petals.

UA3GTs were selected as candidates involved in flavonoid metabolic pathway, $U A 3 G T$ s induced blue color petals in Nymphaea 'King of Siam'. All these results revealed the biochemical and molecular mechanisms of blue color formation and provided the basis for breeding blue hardy waterlily cultivars. This study will improve our understanding of the molecular mechanism of blue flowers and provide the evidence for molecular breeding theory concerning the basis of flower color modifications in ornamental plants.

\section{Conclusions}

The transcript dataset derived by using high-throughput sequencing technology provided an efficient approach for identifying vital unigenes in Nymphaea. Transcriptome analyses from the petals of two different coloring stages of blue tropical waterlily cultivar Nymphaea 'King of Siam' resulted in 112,485 unigenes. 127 unigenes could be identified as putative homologues of color-related genes in other species, including 28 up-regulated and 5 down-regulated unigenes. The composition of flavonoids and the metabolic pathways related to blue formation were identified, and UA3GTs were selected as the most important candidates involved in the flavonoid metabolic pathway, which induced blue petal color formation. This study provides a comprehensive molecular biology background for researches on Nymphaea, particularly with respect to the process of blue color formation.

\section{Additional files}

Additional file 1: Table S1. List of DEGs which related to flavonoid biosynthesis in flower petals of Nymphaea 'King of Siam'. (DOCX 25 kb)

Additional file 2: Table S2. Qualitative analysis of flavonoids in flower petals of Nymphaea 'King of Siam'. (DOCX $21 \mathrm{~kb}$ )

Additional file 3: Table S3. Quantitative analysis of flavonoids in flower petals of Nymphaea 'King of Siam'. (DOCX $18 \mathrm{~kb}$ )

Additional file 4: Table S4. Output statistics of RNA-Seq of Nymphaea 'King of Siam'. (DOCX 17 kb)

Additional file 5: Figure S1. Volcano plot between S1 and S3. (PNG 28 kb)

Additional file 6: Table S5. List of pathway enriched differentially expressed genes in Nymphaea 'King of Siam'. (DOCX 16 kb)

Additional file 7: Figure S2. Correlation of gene expression results respectively obtained by two methods (RT-qPCR analysis and RNA-Seq) for color-related genes in S1 and S3. (TIF $1641 \mathrm{~kb}$ )

Additional file 8: Table S6. List of UA3'GT in Nymphaea 'King of Siam'. (DOCX $17 \mathrm{~kb}$ )

\section{Abbreviations}

ANS: Anthocyanidin synthase; CHI: Chalcone isomerase; CHS: Chalcone synthase; COG: Clusters of Orthologous Groups of proteins; Cy: Cyanidin; Cy3Glc: Cyanidin 3-O-glucoside; DEGs: Differentially expressed genes; DFR: Dihydroflavonol 4-reductase; Dp: Delphinidin; Dp3'galloylacetylGal: Delphinidin 3'-O-(-2"-O-galloyl-6"-O-acetyl- $\beta$-galactopyranoside; Dp3'galloylGal: Delphinidin 3'-O-(-2"-O-galloyl- $\beta$-galactopyranoside; Dp3acetylGlc: Delphinidin 3-O-(-6"-O-acetyl- $\beta$-glucopyranoside; Dp3Gal: Delphinidin 3-O- $\beta$-galactopyranoside; F3'5'H: Flavonoid 3', 5'hydroxylase; F3'H: Flavonoid 3'-hydroxylase; F3H: Flavanone 3-hydroxylase; FLS: Flavonol synthase; GO: Gene Ontology; I-Class UPLC/Xevo TQ MS: I-Class ultra-high performance liquid chromatography/Xevo triple-quadrupole mass spectrometry; KEGG: Kyoto Encyclopedia of Genes and Genomes; KOG: Eukaryotic Orthologous Groups of proteins; N50: Covering 50 \% of all the nucleotide sequences of the largest unigene length; NR: Non-redundant; Pfam: Protein family; Q30 percentage: Percentage of bases with sequencing 
error rate lower than $1 \%$; RNA-Seq: RNA sequencing; RT-qPCR: Real-time quantitative polymerase chain reaction; TA: Total anthocyanins content; TF: Total flavonols content; UA3GT: UDP-glycoside: Anthocyanin 3-Oglycosyltransferase

\section{Funding}

This study was financially supported by the National Natural Science

Foundation of China (Grant No. 31471910).

\section{Availability of data and materials}

The datasets supporting the conclusions of this article are included within the article and its additional files.

\section{Authors' contributions}

QW carried out sequence data analysis and drafted the manuscript. JW contributed to manuscript revision. SSL organized the manuscript. HJZ and RYW assisted with the sample collection. CYF and DDY contributed to oil extraction and RNA extraction. All authors read and approved the final version of the manuscript.

\section{Competing interests}

The authors declare that they have no competing interests.

\section{Consent for publication}

Not applicable.

\section{Ethics approval and consent to participate}

Not applicable

\section{Author details}

'Key Laboratory of Plant Resources and Beijing Botanical Garden, Institute of Botany, Chinese Academy of Sciences, Beijing 100093, China. ${ }^{2}$ University of Chinese Academy of Sciences, Beijing 100049, China. ${ }^{3}$ College of Horticulture, Nanjing Agricultural University, Nanjing 210095, China.

\section{Received: 9 May 2016 Accepted: 28 October 2016}

Published online: 09 November 2016

\section{References}

1. Weiss MR. Floral color change: a widespread functional convergence. Am J Bot. 1995:82:167-85.

2. Tanaka Y, Sasaki N, Ohmiya A. Biosynthesis of plant pigments: anthocyanins, betalains and carotenoids. Plant J. 2008:54:733-49.

3. Tanaka Y, Tsuda S, Kusumi T. Metabolic engineering to modify flower color. Plant Cell Physiol. 1998;39:1119-26.

4. Iwashina T. The structure and distribution of the flavonoids in plants. J Plant Res. 2000:113:287-99.

5. Chandler S, Tanaka Y. Genetic modification in floriculture. Crit Rev Plant Sci. 2007:26:169-97.

6. Yang RZ, Wei XL, Gao FF, Wang LS, Zhang HJ, Xu YJ, Li CH, Ge YX, Zhang JJ, Zhang J. Simultaneous analysis of anthocyanins and flavonols in petals of lotus (Nelumbo) cultivars by high-performance liquid chromatography-photodiode array detection/electrospray ionization mass spectrometry. J Chromatogr A. 2009:1216:106-12.

7. Sasaki N, Nakayama T. Achievements and perspectives in biochemistry concerning anthocyanin modification for blue flower coloration. Plant Cell Physiol. 2015;56:28-40

8. Borsch T, Hilu KW, Wiersema JH, Löhne C, Barthlott W, Wilde V. Phylogeny of Nymphaea (Nymphaeaceae): Evidence from substitutions and microstructural changes in the chloroplast trnT-trnF region. Int J Plant Sci. 2007;168:639-71

9. Yin DD, Yuan RY, Wu Q, Li SS, Shao S, Xu YJ, Hao XH, Wang LS. Assessment of flavonoids and volatile compounds in tea infusions of water lily flowers and their antioxidant activities. Food Chem. 2015;187:20-8.

10. Yildirim $A B$, Karakas FP, Turker AU. In vitro antibacterial and antitumor activities of some medicinal plant extracts, growing in Turkey. Asian Pac J Trop Med. 2013:6:616-24

11. Parimala M, Shoba FG. In vitro antimicrobial activity and HPTLC analysis of hydroalcoholic seed extract of Nymphaea nouchali Burm. f. BMC Complement Altern Med. 2014;14:361
12. Zhang Z, ElSohly HN, Li XC, Khan SI, Broedel SE, Raulli RE, Cihlar RL, Burandt C. Walker LA. Phenolic Compounds from Nymphaea odorata. J Nat Prod. 2003;66:548-50

13. Dhanabal SP, Raja MKMM, Ramanathan M, Suresh B. Hypoglycemic activity of Nymphaea stellata leaves ethanolic extract in alloxan induced diabetic rats. Fitoterapia. 2007;78:288-91.

14. Rajagopal K, Sasikala K. Antidiabetic activity of hydro-ethanolic extracts of Nymphaea Stellata flowers in normal and alloxan induced diabetic rats. Afr J Pharm Pharmacol. 2008:2:173-8.

15. Bing SJ, Ha D, Kim MJ, Park E, Ahn G, Kim DS, Ko RK, Park JW, Lee NH, Jee Y. Geraniin down regulates gamma radiation-induced apoptosis by suppressing DNA damage. Food Chem Toxicol. 2013;57:147-53.

16. Thippeswamy B, Mishra B, Veerapur V, Gupta G. Anxiolytic activity of Nymphaea alba Linn. in mice as experimental models of anxiety. Indian J Pharm. 2011:43:50-5.

17. Bhandarkar MR, Khan A. Antihepatotoxic effect of Nymphaea stellata willd. against carbon tetrachloride-induced hepatic damage in albino rats J Ethnopharmacol. 2004;91:61-4.

18. Huang GZ, Deng HQ, Li ZX, Li G. Water lily. Beijing: China Forestry Publishing House; 2009.

19. Speichert CG, Speichert S, Lovejoy A. Encyclopedia of water garden plants. Oregon: Timber Press Portland; 2004.

20. Songpanich P. Hongtrakul V. Intersubgeneric cross in Nymphaea spp. L. to develop a blue hardy waterlily. Sci Hortic. 2010;124:475-81.

21. Lou Q, Liu YL, Qi YY, Jiao SZ, Tian FF, Jiang L, Wang YJ. Transcriptome sequencing and metabolite analysis reveals the role of delphinidin metabolism in flower colour in grape hyacinth. J Exp Bot. 2014;65:3157-64.

22. Zhao DQ, Jiang Y, Ning CL, Meng JS, Lin SS, Ding W, Tao J. Transcriptome sequencing of a chimaera reveals coordinated expression of anthocyanin biosynthetic genes mediating yellow formation in herbaceous peony (Paeonia lactiflora Pall.). BMC Genomics. 2014;15:689.

23. Zhang YZ, Cheng YW, Ya HY, Xu SZ, Han JM. Transcriptome sequencing of purple petal spot region in tree peony reveals differentially expressed anthocyanin structural genes. Front Plant Sci. 2015:6:964.

24. Wang Z, Gerstein M, Snyder M. RNA-Seq: a revolutionary tool for transcriptomics. Front Plant Sci. 2009:10:57-63.

25. Zhu ML, Zheng XC, Shu QY, Li H, Zhong PX, Zhang HJ, Xu YJ, Wang LJ, Wang LS. Relationship between the composition of flavonoids and flower colors variation in tropical water lily (Nymphaea) cultivars. PLoS One. 2012;7:e34335.

26. Grabherr MG, Haas BJ, Yassour M, Levin JZ, Thompson DA, Amit I, Adiconis $X$, Fan L, Raychowdhury R, Zeng QD, et al. Full-length transcriptome assembly from RNA-Seq data without a reference genome. Nat Biotechnol. 2011;29:644-52.

27. Haas BJ, Papanicolaou A, Yassour M, Grabherr M, Blood PD, Bowden J, Couger MB, Eccles D, Li B, Lieber M, et al. De novo transcript sequence reconstruction from RNA-seq using the Trinity platform for reference generation and analysis. Nat Protoc. 2013;8:1494-512

28. Langmead B, Trapnell C, Pop M, Salzberg SL. Ultrafast and memory-efficient alignment of short DNA sequences to the human genome. Genome Biol. 2009;10:R25

29. Li B, Dewey CN. RSEM: accurate transcript quantification from RNA-Seq data with or without a reference genome. BMC Bioinformatics. 2011:12:323.

30. Trapnell C, Williams BA, Pertea G, Mortazavi A, Kwan G, van Baren MJ, Salzberg SL, Wold BJ, Pachter L. Transcript assembly and quantification by RNA-Seq reveals unannotated transcripts and isoform switching during cell differentiation. Nat Biotechnol. 2010;28:511-5.

31. Anders S, Huber W. Differential expression analysis for sequence count data. Genome Biol. 2010;11:R106.

32. Luo HL, Chen SM, Wan HJ, Chen FD, Gu CS, Liu ZL. Candidate reference genes for gene expression studies in water lily. Anal Biochem 2010:404:100-2

33. Feng $\mathrm{C}$, Chen $\mathrm{M}, \mathrm{Xu} \mathrm{CJ}$, Bai L, Yin XR, Li X, Allan AC, Ferguson IB, Chen KS. Transcriptomic analysis of Chinese bayberry (Myrica rubra) fruit development and ripening using RNA-Seq. BMC Genomics. 2012;13:19.

34. Hong $Y$, Tang $X J$, Huang $H$, Zhang $Y$, Dai SL. Transcriptomic analyses reveal species-specific light-induced anthocyanin biosynthesis in chrysanthemum. BMC Genomics. 2015:16:202

35. Debnath S, Ghosh S, Hazra B. Inhibitory effect of Nymphaea pubescens Willd. flower extract on carrageenan-induced inflammation and $\mathrm{CCl}_{4}$-induced hepatotoxicity in rats. Food Chem Toxicol. 2013;59:485-91. 
36. Hsu CL, Fang SC, Yen GC. Anti-inflammatory effects of phenolic compounds isolated from the flowers of Nymphaea mexicana Zucc. Food Funct. 2013:4: 1216-22.

37. Tanaka Y. Flower colour and cytochromes P450. Phytochem Rev. 2006;5: 283-91.

38. Chandler SF, Brugliera F. Genetic modification in floriculture. Biotechnol Lett. 2011:33:207-14

39. Katsumoto $Y$, Fukuchi-Mizutani M, Fukui $Y$, Brugliera F, Holton TA, Karan M, Nakamura N, Yonekura-Sakakibara K, Togami J, Pigeaire A, et al. Engineering of the rose flavonoid biosynthetic pathway successfully generated blue-hued flowers accumulating delphinidin. Plant Cell Physiol. 2007:48:1589-600

40. Brugliera F, Tao GQ, Tems U, Kalc G, Mouradova E, Price K, Stevenson K, Nakamura N, Stacey I, Katsumoto Y. Violet/blue chrysanthemums-metabolic engineering of the anthocyanin biosynthetic pathway results in novel petal colors. Plant Cell Physiol. 2013;54:1696-710.

41. Noda N, Aida R, Kishimoto S, Ishiguro K, Fukuchi-Mizutani M, Tanaka Y, Ohmiya A. Genetic engineering of novel bluer-colored chrysanthemums produced by accumulation of delphinidin-based anthocyanins. Plant Cell Physiol. 2013;54:1684-95.

42. Johnson ET, Ryu S, Yi HK, Shin B, Cheong H, Choi G. Alteration of a single amino acid changes the substrate specificity of dihydroflavonol 4-reductase. Plant J. 2001;25:325-33.

43. Grotewold E. The genetics and biochemistry of floral pigments. Annu Rev Plant Biol. 2006;57:761-80.

44. Fossen T, Frøystein NÅ, Andersen ØM. Myricetin 3-rhamnosyl (1 $\rightarrow 6)$ galactoside from Nymphaéa x marliacea. Phytochemistry. 1998;49:1997-2000.

45. Fossen T, Anderson ØM. Delphinidin 3'-galloylgalactosides from blue flowers of Nymphae'a caerulea. Phytochemistry. 1999;50:1185-8.

46. Fossen T, Larsen $\AA$, Kiremire BT, Andersen ØM. Flavonoids from blue flowers of Nymphaea caerulea. Phytochemistry. 1999;51:1133-7.

47. Zhu ML, Wang LS, Zhang HJ, Xu YJ, Zheng XC, Wang LJ. Relationship between the composition of anthocyanins and flower color variation in hardy water lily (Nymphaea spp.) cultivars. Chin Bull Bot. 2012;47:437-53.

48. Fukuchi-Mizutani M, Okuhara H, Fukui $Y$, Nakao M, Katsumoto $Y$, Yonekura-Sakakibara K, Kusumi T, Hase T, Tanaka Y. Biochemical and molecular characterization of a novel UDP-glucose: anthocyanin 3'-O-glucosyltransferase, a key enzyme for blue anthocyanin biosynthesis, from gentian. Plant Physiol. 2003;132:1652-63.

49. Kogawa K, Kato N, Kazuma K, Noda N, Suzuki M. Purification and characterization of UDP-glucose: anthocyanin 3', 5'-O-glucosyltransferase from Clitoria ternatea. Planta. 2007;226:1501-9.

50. Ogata J, Kanno Y, Itoh Y, Tsugawa H, Suzuki M. Plant biochemistry: anthocyanin biosynthesis in roses. Nature. 2005;435:757-8.

\section{Submit your next manuscript to BioMed Central and we will help you at every step:}

- We accept pre-submission inquiries

- Our selector tool helps you to find the most relevant journal

- We provide round the clock customer support

- Convenient online submission

- Thorough peer review

- Inclusion in PubMed and all major indexing services

- Maximum visibility for your research

Submit your manuscript at www.biomedcentral.com/submit

) Biomed Central 